\title{
Development of inhalable cubosome nanoparticles of Nystatin for effective management of Invasive Pulmonary Aspergillosis
}

\author{
Marzuka Kazi ${ }^{1}$, Mohamed Hassan Dehghan¹ \\ 'Y.B. Chavan College of Pharmacy, Department of Pharmaceutics, Dr. Rafiq Zakaria Campus,Aurangabad, India
}

ORCID IDs of the authors: M.K. 0000-0002-6103-7806; M.H.D. 0000-0002-8082-9454

Cite this article as: Kazi, M., \& Dehghan M. H. (2020). Development of inhalable cubosome nanoparticles of Nystatin for effective management of Invasive Pulmonary Aspergillosis. Istanbul Journal of Pharmacy, 50 (3), 224-237.

\begin{abstract}
Background and Aims: Invasive pulmonary aspergillosis (IPA) is an imperative concern in the present era due to its high occurrence and mortality rate in severely immunocompromised patients. The present study was designed to develop, optimize and characterize encapsulated nystatin (NYS) cubosome nanoparticles as an inhalable system, a viable alternative for effective management of IPA.

Methods: A dry lipidic film comprising glycerol monooleate (GMO), Span 83, Poloxamer (P-407) and dispersed NYS was subjected to ultrasound sonication to produce colloidal dispersion of cubosomes. The process and formulation variables were screened using Plackett Burman design and further optimized by Box Behnken design by evaluating its effect on particle size, polydispersity index (PDI), zeta potential and entrapment efficiency.

Results: The optimized NYS cubosomes were nearly spherical with some irregular polyangular symmetry as visualized by transmission electron microscopy (TEM). Further, small angle X-ray scattering (SAXS) affirmed Pn $3 \mathrm{~m}$ cubic mesophasic structure. The optimized nanoparticles had particle size $263.5 \mathrm{~nm}$, zeta potential $-14.4 \mathrm{mV}$, PDI 0.283 and entrapment efficiency $82 \%$. The in-vitro cytotoxicity assay indicated that NYS cubosomes reduced cell cytotoxicity in contrast to pure drug post $48 \mathrm{~h}$. In-vitro haemolytic assay denoted lower toxicity of formulation as compared to free drug. In-vitro drug release studies highlighted, slow but continuous release from NYS cubosomes until 48h and showcased Higuchi release kinetics. Likewise, NYS cubosome demonstrated higher antifungal activity compared to drug suspended in phosphate buffer.

Conclusion: Thus, non-invasive feature and contemplated target specificity of nystatin loaded cubosome nanoparticles pave a mode for its prospect as pulmonary delivery to combat IPA.

Keywords: Nystatin, cubosome nanoparticles, aspergillosis, drug design, pulmonary
\end{abstract}

\section{INTRODUCTION}

Lipidic, polymeric and polyelectrolyte complex based nanoparticulate drug delivery lately has been widely studied as potential carriers for delivery of numerous drug candidates such as anticancer (Chishti, Jagwani, Dhamecha, Jalalpure, \& Dehghan, 2019), peptide (on Halling Laier et al., 2018; Poddar \& Sawant, 2017), antifungals (Furedi et al., 2017), antivirals (Mandal, Prathipati, Belshan, \& Destache, 2019) and antibacterials (Carneiro et al., 2019; Rani et al., 2018). The prevalence rate of invasive pulmonary aspergillosis (IPA) is high in severely immunocompromised patients suffering from HIV, cancer, critically ill patients, organ transplant patients and in individuals with known history of chronic obstructive pulmonary disease (Szalewski, Hinrichs, Zinniel, \& Barletta, 2018). The management of IPA still remains a challenge as most of the antifungal used in its treatment present resistance or pose a high toxicity profiles (Kosmidis \& Muldoon, 2017). Nystatin (NYS) a broad 
spectrum is effective against azole resistant and amphotericin B resistant strains of Candida albicans (Offner et al., 2004). Concentration dependent activity of NYS is key-in factor to rapid onset of action for successful treatment. Additionally, NYS is known to showcase long post-antifungal effect, thus justifies the rational for reduction in its frequency of administration (Marin-Quintero et al., 2013; Ryder, 1999). The formulation challenge revealed by NYS as a result of low solubility and low permeability, is the prime reason that makes it complicated to design a controlled release delivery for this drug (Fernandez Campos, Calpena Campmany, Rodriguez Delgado, Lopez Serrano, \& Clares Naveros, 2012). Researchers have tried formulating NYS in various delivery systems such as lipid intravenous emulsion (Ryder, 1999), nanoemulsions (Fernandez Campos et al., 2012), micellar gels (Maqsood et al., 2015), mucoadhesives for topical use (Sakeer, Al-Zein, Hassan, Desai, \& Nokhodchi, 2010), liposomes (Khan, Faisal, \& Mohammad, 2006), niosomes (El-Ridy, Abdelbary, Essam, El-Salam, \& Kassem, 2011), microparticles (Kim, Son, \& Kwon, 2018), pastilles (Silva et al., 2017), pellets and drug particulates system in toothpaste (Pinto Reis, Vasques Roque, Baptista, \& Rijo, 2016) to solve drug related problems.

Nano-particulate formulations as new frontiers are being developed to improve treatment outcomes in IPA patients. Cubosomes, a dispersion of the bicontinuous cubic mesophase have contorted lipid bilayer as their basic unit and two extremely tortuous non-intersecting water channels (Esposito et al., 2005). The high lipidic content and large surface area posed by these structures makes them desirable carriers for efficient encapsulation of hydrophilic, hydrophobic, or amphiphilic drug molecules (Shi et al., 2017). Biocompatibility, biodegradability, bioadhesion and prolonged drug release ability interpret cubosomes to be an effective carrier for IPA drug therapy (Madheswaran, Kandasamy, Bose, \& Karuppagounder, 2019). Moreover, the nanoparticle size range of these structures facilitates passive targeting to pulmonary route, and leads to deposition of the drug to the most sorted areas of the lower respiratory tract or into the deep regions of lungs (Newman, 2017). In case of pulmonary infection, delivering the drug to the target site of action is beneficial in reducing drug level in the systemic circulation although the desired therapeutic index at the site of infection is maintained (Islam \& Ferro, 2016). This route of administration helps to reduce the drug related toxicity in comparison to the parenteral route (Das et al., 2015).

On the basis of these considerations, the research objective was to overcome formulation challenges presented by the drug and in conception of a site-specific delivery system, so as to achieve enhanced effectiveness. The present study was designed to develop, optimize and characterize encapsulated Nystatin cubosome nanoparticles for effective management of IPA, to be targeted through inhalable pulmonary route.

\section{MATERIALS AND METHODS}

Nystatin (NYS), Polaxamer 407 (P-407) was a kind gift sample from Glenmark Pharmaceuticals, (Mumbai, India). Dimodan HPM-90 (solid grade GMO) and Cithrol GMO-HP-SO-LK was sorted as a gift sample obtained from Dupont (Gurgaon, India) and Croda, (Mumbai, India) respectively. Span 80 and Span 83 was a generously donated by Cipla Pvt. Ltd., (Mumbai, India). Organic solvents like dimethyl sulfoxide, methanol, dimethyl formaide, chloroform were purchased from Loba chemie, Mumbai whereas dextrose, peptone and agar were procured from Fisher scientific, India. Aspergillus fumigatus (NCIM 902) was procured from National chemical laboratory, Pune. Dialysis bag with a molecular weight cut-off (MWCO) of 3,500 Da was purchased from Merck Millipore, India, Pvt. Ltd. (Bengaluru).

\section{Ishikawa Fishbone diagram and risk assessment by failure mode and effects analysis (FMEA)}

Risk assessment for the material attributes and process variables was carried out after analyzing the available literature, in order to estimate its effect on critical quality attributes (CQAs) viz., particle size (prediction of inhalation through nasopulmonary route and its absorption potential in lungs), drug loading and entrapment efficiency (to predict available drug for absorption, release kinetics of drug). Ishikawa or Fishbone presentation is a cause and effect type of diagram that was established in order to find all the possible variables that could have an impact on CQAs of the drug product i.e. NYS loaded cubosomes shown on the primary arrow (central horizontal arrow i.e. the studied effect is "the fish head"). The secondary arrows (vertical slanting arrows i.e. the potential causes and subcauses define the "fish bone structure") represent the process and formulation variables e.g. materials, people, cubosome formation process and instrument. The tertiary arrows represent the factors in-return impacting these process and formulation variables e.g. sonication, lyophilizer, surfactant, organic solvent, drug etc. Thus, the use of the Ishikawa diagram helps illustrate, identify and analyze all the factors connected to the main aim of the study. The factors marked in the red text have been studied in the present work. The quality target product profile (QTPP) was identified as dosage form, its shape, its type, strength, route of administration.

\section{Plackett Burman experimental design}

Based on preliminary trial results and literature survey potential factors which could impact the critical attributes of the final product was identified. Screening of process variables and formulation components was essentially studied using Plackett Burman design (Table 1) to understand its effect on critical qual-

\section{Table 1. Factors and their levels in Plackett Burman Experimental design.}

\begin{tabular}{|c|c|c|}
\hline \multirow{2}{*}{ Factors } & \multicolumn{2}{|c|}{ Levels } \\
\hline & Low & High \\
\hline$X_{1}:$ NYS concentration & $1.23 \%$ & $2.23 \%$ \\
\hline $\mathrm{X}_{2}$ : Type of GMO & HPM-90 & HP-SO-LK \\
\hline $\mathrm{X}_{3}$ : Type of high HLB surfactant & Span 80 & Span 83 \\
\hline $\mathrm{X}_{4}$ : Type of low HLB surfactant & P 188 & P 407 \\
\hline $\begin{array}{c}\mathrm{X}_{5} \text { : Concentration of high HLB } \\
\text { surfactant }(\mathrm{mg})\end{array}$ & 150 & 300 \\
\hline $\begin{array}{c}\mathrm{X}_{6}: \text { Concentration of low HLB } \\
\text { surfactant }(\mathrm{mg})\end{array}$ & 100 & 200 \\
\hline $\mathrm{X}_{7}$ : Sonication time $(\min )$ & 15 & 30 \\
\hline $\mathrm{X}_{8}:$ pulse rate & $3 / 7 \mathrm{sec}$ & $9 / 18 \mathrm{sec}$ \\
\hline
\end{tabular}


ity attributes (CQAs). Accordingly, the study was undertaken for 8 factors at 2 levels and 12 experimental runs were conducted.

NYS-loaded cubosomes were prepared through a modified coarse method (Ali, Noguchi, Iwao, Oka, \& Itai, 2016; Bei, Marszalek, \& Youan, 2009). Briefly, for each batch, different concentration of GMO (600 mg), Poloxamer and Span as in Table 2 was placed in a $100 \mathrm{ml}$ round bottom flask. Further chloroform $(5 \mathrm{ml})$ was added to aid complete solubilization of lipids and surfactant so as to establish a one-phase system. Subsequently, a second phase comprising of Nystatin dissolved in DMSO $(0.5-1 \mathrm{ml})$, was added to the above lipidic surfactant system. Chloroform was allowed to evaporate in a rotatory evapora-

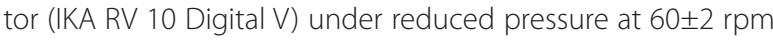
and a temperature of $60 \pm 2^{\circ} \mathrm{C}$, leading to the formation of a thin film at the bottom of the flask. A volume of $50 \mathrm{ml}$ of PBS buffer saline ( $\mathrm{pH}=7.4)$ was added to the dry lipidic drug film to form coarse dispersions by the aid of a probe sonicator (Ali et al., 2016) (Sonics Vibra cell VCX 500) at $25^{\circ} \mathrm{C}, 70 \%$ amplitude with varying the sonication time and pulse rate as indicated in Table 2. The resulting cubosome colloidal dispersions were microcentrifuge (Remi motors, RM 12-C) at 12000 rpm for 30 mins with repeated washings to remove the unentrapped drug, and were stored in a vial at R.T $\left(25^{\circ} \mathrm{C}\right)$ until further evaluation.

The implications of the model and factor coefficients were analyzed by undertaking multi-linear regression analysis and analysis of variance. Experimental checks were taken in triplicate. The dependent variables (CQAs) were identified as average particle size $\left(Y_{1}\right)$, zeta potential $\left(Y_{2}\right)$, entrapment efficiency $\left(Y_{3}\right)$ and polydispersity index $\left(\mathrm{Y}_{4}\right)$ and in-vitro antifungal activity $\left(\mathrm{Y}_{5}\right)$.

\section{Optimization of NYS cubosomes using Box Beknhen (BB) design}

After studying Plackett Burman design for 8 factors at 2 levels, the cubosomes were optimized for process and formulation variables using Box Beknhen design. Factors that were fixed are as follows GMO grade was selected as HP-SO-LK, low HLB surfactant was preferred to be Span 83 whereas high HLB surfactant was chosen to be poloxamer 407, the pulse rate was set to be $3 / 7 \mathrm{sec}$. The concentration of drug and GMO was fixed to $1.23 \% \mathrm{w} / \mathrm{w}$ and $600 \mathrm{mg}$. The factors such as the concentration of high HLB surfactant, low HLB surfactant and probe sonication time were rigidly studied at 3 levels in BB design (Table 3) for further optimization of NYS cubosomes. The randomized experimental designed batches were formulated (Table 4) as per the above-mentioned procedure and statistically analyzed by Design expert ${ }^{\circledR 7}$ software.

Table 3. Factors and their levels set in Box Behnken Experimental design.

\begin{tabular}{|cccc|}
\hline \multirow{2}{*}{ Factors } & \multicolumn{3}{c|}{ Levels } \\
\cline { 2 - 4 } & - & $\mathbf{0}$ & + \\
\hline $\mathrm{X}_{1}$ : concentration of Low HLB & 150 & 225 & 300 \\
$\begin{array}{c}\text { surfactant } \\
\mathrm{X}_{2} \text { : concentration of High HLB } \\
\text { surfactant }\end{array}$ & 50 & 100 & 150 \\
$\mathrm{X}_{3}$ : sonication time & 10 & 15 & 20 \\
\hline
\end{tabular}

Table 4. Box Behnken Design of Experiment.

\begin{tabular}{|cccc|} 
Formulation & $\mathbf{X}_{\mathbf{1}}(\mathbf{m g})$ & $\mathbf{X}_{\mathbf{2}}(\mathbf{m g})$ & $\mathbf{X}_{\mathbf{3}}$ (mins) \\
\hline B1 & 225 & 100 & 15 \\
B2 & 225 & 150 & 10 \\
B3 & 225 & 50 & 10 \\
B4 & 150 & 100 & 20 \\
B5 & 225 & 100 & 15 \\
B6 & 225 & 50 & 20 \\
B7 & 225 & 100 & 15 \\
B8 & 150 & 150 & 15 \\
B9 & 225 & 150 & 20 \\
B10 & 300 & 150 & 15 \\
B11 & 150 & 150 & 15 \\
B12 & 300 & 100 & 20 \\
B13 & 225 & 100 & 15 \\
B14 & 225 & 100 & 15 \\
B15 & 300 & 100 & 10 \\
B16 & 300 & 50 & 15 \\
B17 & 150 & 100 & 10 \\
\hline
\end{tabular}

Table 2. Plackett Burman Design of Experiment.

\begin{tabular}{cccccccccc}
\hline Formulation & $\mathbf{X}_{\mathbf{1}}(\%)$ & $\mathbf{X}_{\mathbf{2}}$ & $\mathbf{X}_{\mathbf{3}}(\%)$ & $\mathbf{X}_{\mathbf{4}}$ & $\mathbf{X}_{\mathbf{5}}(\mathbf{m g})$ & $\mathbf{X}_{\mathbf{6}}(\mathbf{m g})$ & $\mathbf{X}_{\mathbf{7}}(\mathbf{m i n})$ & $\mathbf{X}_{\mathbf{8}}(\mathbf{r p m})$ \\
\hline D1 & 1.23 & HPM-90 & Span 80 & P-188 & 150 & 100 & 15 & $3 / 7$ \\
D2 & 1.23 & HP-SO-LK & Span 83 & P-188 & 300 & 200 & 30 & $3 / 7$ \\
D3 & 2.23 & HPM-90 & Span 83 & P-407 & 300 & 100 & 15 & $3 / 7$ \\
D4 & 1.23 & HP-SO-LK & Span 83 & P-407 & 150 & 100 & 15 & $9 / 18$ \\
D5 & 1.23 & HPM-90 & Span 83 & P-188 & 300 & 200 & 15 & $9 / 18$ \\
D6 & 2.23 & HPM-90 & Span 80 & P-188 & 300 & 100 & 30 & $9 / 18$ \\
D7 & 2.23 & HPM-90 & Span 83 & P-407 & 150 & 200 & 30 & $9 / 18$ \\
D8 & 1.23 & HP-SO-LK & Span 80 & P-407 & 300 & 100 & 30 & $9 / 18$ \\
D9 & 2.23 & HP-SO-LK & Span 80 & P-188 & 150 & 200 & 15 & $9 / 18$ \\
D10 & 1.23 & HPM-90 & Span 80 & P-407 & 150 & 200 & 30 & $3 / 7$ \\
D11 & 2.23 & HP-S0-LK & Span 83 & P-188 & 150 & 100 & 30 & $3 / 7$ \\
D12 & 2.23 & HP-S0-LK & Span 80 & P-407 & 300 & 200 & 15 & $3 / 7$ \\
\hline
\end{tabular}




\section{Characterization}

Particle size, zeta potential and poly dispersibility index The mean size and size distribution of colloidal particles is measured using Malvern Zetasizer Nano Series (Malvern Instruments, Malvern, India). Prior to the examination, samples were diluted with purified water to a scattering intensity of about 150-300 kcps and the dispersant viscosity was set to $0.8872 \mathrm{CP}$ at $25^{\circ} \mathrm{C}$. The size (z-average), polydispersity index (PDI) and zeta potential was determined in triplicate.

\section{Polarized light microscopy (PLM)}

PLM (Carl Zeiss, Jena, Germany) imaging was performed so as to ascertain the gross morphology of the formed nanoparticle at $25^{\circ} \mathrm{C}$ i.e. cubosome or hexosome.

\section{Encapsulation efficiency}

Samples from each batch were centrifuged using microcentrifuge (Remi motors, RM 12-LC) at 12,000 rpm for $30 \mathrm{~min}$. Aliquots from the supernatant of each batch were diluted using 5\% Triton-X solution in PBS (pH 7.4) to disrupt any lipidic fragment (Rizwan et al., 2011). The entrapped drug amount was calculated after analysis of the drug concentration in the diluted supernatant using UV spectrophotometer (UV 1800, Shimadzu Japan) at max $306 \mathrm{~nm}$.

EE was calculated using the following equation:

$$
E E \%=\frac{C(\text { total drug concentration })-C(\text { free drug })}{C(\text { total drug concentration })} \times 100(\text { Eq. 1) }
$$

\section{In-vitro antifungal activity}

The in-vitro antifungal activity of the optimized colloidal dispersion was evaluated against Aspergillus fumigatus (NCIM 902). Inoculum suspensions of Aspergillus fumigatus were prepared from fresh, mature (3-5 day old) cultures grown on Sabouraud dextrose agar slants. The colonies were covered with $5 \mathrm{ml}$ of distilled sterile water. Tween 20 (1\%) was added to facilitate the preparation of Aspergillus inocula. The inocula were achieved by carefully rubbing the colonies with a sterile loop; the isolates were then shaken vigorously for $15 \mathrm{~s}$ with a vortex mixer and then transferred to a sterile tube. The optical density of the suspensions was measured by UV spectrophotometer (Shimadzu UV-1600) was set as 0.13 at $530 \mathrm{~nm}$ (Petrikkou et al., 2001). Agar well diffusion method was used to evaluate antifungal activity, $1 \mathrm{ml}$ of microbial inoculum was seeded into SDA medium and poured into petri plates, a sterile cork borer of diameter $6 \mathrm{~mm}$ were punched aseptically to create a well and colloidal dispersion $(0.1 \mathrm{ml})$ is added into the well. The plates were incubated for $48 \mathrm{hrs}, 27^{\circ} \mathrm{C}$ (Aspergillus fumigatus).

\section{In-vitro drug release}

The in-vitro release profiles of NYS-loaded cubosomes were evaluated using a membrane dialysis method (Yang et al., 2012). Optimized cubosome formulation $(2 \mathrm{ml}$ ) was dispersed in $1 \mathrm{ml}$ of release medium (phosphate buffer $\mathrm{pH}$ 7.4) consisting of $0.25 \% \mathrm{w} / \mathrm{v}$ of sodium lauryl sulphate. The resulting dispersion was added to the dialysis bag (MWCO 3,500 Da) and sealed. The dialysis bag was immersed in beaker containing
$10 \mathrm{ml}$ of release medium placed over a magnetic stirrer (Remi Equipment, Mumbai, India), maintained at $37^{\circ} \mathrm{C}$ and set at 100 rpm. At specified time intervals of 1, 2, 4, 6, 8, 12 and $24 \mathrm{~h}$ on the first day followed by $24 \mathrm{~h}$ interval for the subsequent day's samples were withdrawn and replaced with same volume of release medium. NYS content in the release medium was assayed using the UV spectroscopy method as used in encapsulation efficiency determination.

\section{Cytotoxicity assays}

The MTT (3-(4,5-dimethylthiazolyl-2-yl)-2,5-diphenyl-tetrazoliumbromide) colorimetric assay was employed to assess the cytotoxicity of NYS and optimized NYS cubosome formulation on A549 cell lines (Adhikari, 2017; Lestner et al., 2010). Cells were seeded in 96-wellplates at a density of $1 \times 10^{5}$ cells/well suspended in $100 \mu$ l of Dulbecco's modified Eagle's medium (DMEM, HiMedia, Nashik, India). Following incubation for 24 $h$, the medium was aspirated from all wells and replaced with $100 \mu \mathrm{l} /$ well culture medium with NYS or NYS cubosome formulation $(3.125,6.125,12.25,25,50,100,150,200 \mu \mathrm{g} / \mathrm{ml})$ and incubated for further $24 \mathrm{~h}$. Cell viability was found by adding $200 \mu \mathrm{l}$ of a $1.25 \mathrm{mg} / \mathrm{ml}$ MTT (Invitrogen, USA) to all well, trail by $4 \mathrm{~h}$ of incubation. Post incubation, MTT and medium were aspirated; formazan product was solubilized in $200 \mu \mathrm{LMSO}$. Multi-detection microplate reader (Biohit BP 800, Helsinki, Finland) was used to measure absorbance at $570 \mathrm{~nm}$. IC50 values were determined for both NYS and optimized NYS cubosomes.

\section{In-vitro hemolysis assay}

Fresh sheep red blood cells (SRBC) obtained from local slaughter house were suspended in sterile saline $5 \% \mathrm{v} / \mathrm{v}$ and washed twice with saline by centrifugation at $1,800 \mathrm{~g}$ for $10 \mathrm{~min}$ (Chuealee, Aramwit, Noipha, \& Srichana, 2011). $0.5 \mathrm{ml}$ of serially diluted NYS or optimized NYS cubosomes having concentrations in the range of $10-500 \mu \mathrm{g} / \mathrm{ml}$ were suspended in $4.5 \mathrm{ml}$ of the washed SRBC having a final hematocrit of $1 \%$. Subsequent to incubation $\left(37^{\circ} \mathrm{C}\right.$ for $\left.1 \mathrm{~h}\right)$, the tube was centrifuged $(1,800 \mathrm{~g}$, $10 \mathrm{~min}$ ), the unlysed cells were removed and hemolysis in the supernatant was estimated by measuring absorbance at 540 $\mathrm{nm}$. No lysis was observed in sterile saline used as negative control whereas $100 \%$ lysis was seen in positive control (1\% Triton $\mathrm{X}-100)$. $\mathrm{HC}_{50}$ value was found for both NYS and optimized formulation.

Hemolysis \% $=\frac{(O D \text { of sample }-O D \text { of negative control) }}{(O D \text { of positive control }- \text { OD of negative control) }} \times 100$ (Eq. 2)

\section{Small angle X-ray scattering (SAXS)}

The SAXS measurements were carried out using Xeuss 2 (Xenocs SAS, France), which consisted of an Eiger $\mathrm{R} 1 \mathrm{M}$ as the detector, GeniX3D Cu 30 Watts Cu tube with 50 KV 0.6 mA current, $1.54 \AA$ as the radiation wavelength, and the scattering angle ranged as $0.2^{\circ}$ to $2.8^{\circ}$. The measurements were taken in a vacuum $\left(25 \pm 0.1^{\circ} \mathrm{C}\right)$. The diffraction patterns acquired were converted to plots of intensity vs. q-value, which facilitated the identifications of the peak point, and its conversion to Miller Indices to recognize the phase structure using SAXS processing software [Scatter developed by S. Förster (University of Hamburg), and L. Apostol (DUBBLE/ESRF)]. 


\section{Differential scanning calorimeter (DSC)}

The physical state of NYS encapsulated in cubic nanoparticles was characterized by DSC thermal analyzer (DSC 60 plus, Shimadzu, Japan). Approximately 5-10 mg of samples including pure NYS, P-407, GMO and optimized NYS cubosomes were placed in a standard aluminum pan and an empty hermetic pan was used as a reference. After purging with pure nitrogen at a flow rate of $10 \mathrm{~mL} / \mathrm{min}$, the samples were analyzed at a heating rate of $10^{\circ} \mathrm{C} / \mathrm{min}$ from $40^{\circ} \mathrm{C}$ to $300^{\circ} \mathrm{C}$.

\section{Fourier transformer infrared spectroscopy (FTIR)}

The physical state of NYS encapsulated in cubic nanoparticles was also characterized by FTIR (IRAffinity-1S, Shimadzu, Japan). Approximately 10-20 mg of samples were compressed to prepare circular $\mathrm{KBr}$ disc of GMO, P-407, Span 83, NYS, optimized cubosome formulation were screened in the range of 400 to $4000 \mathrm{~cm}^{-1}$.

\section{X-ray diffractometer}

XRD experiments were carried out on a PW3040/60 X'pert PRO (PANalytical, Netherlands). Cu X-ray tube operating at $45 \mathrm{kV}$ and $40 \mathrm{~mA}$ produced incident $\mathrm{X}$-radiation. Diffraction images were obtained on an X'Pert data collector. Samples such as NYS and optimized NYS cubosome formulation were analysed at $25^{\circ} \mathrm{C}$ over the $2 \mathrm{q}$ range $2-80^{\circ}$ with a step size of $0.05^{\circ}$ (60 s).

Field emission gun transmission electron microscope (FEG TEM)

The samples were prepared by negatively staining in $1 \%(\mathrm{~W} / \mathrm{V})$ phospotungstic acid for 3-5 min. A 5- $\mu$ l droplet of the cubo- some suspension was placed onto a 300 mesh carbon coated copper grid, and was allowed to settle for 3-5 min. Next, the excess fluid was removed by absorbent paper. Thereafter visualized on a FEl Model Tecnai G2, F30 300KV (FEl-USA) and snapped on a Gatan axis-mount 2k×2k digital camera.

\section{RESULTS AND DISSCUSSION}

\section{Risk estimation: Ishikawa diagram}

The fishborne diagram was used to estimate the potential cause and effect correlation of process and formulation variable for development of inhalable cubosomes. CQAs such as particle size, PDI, zeta potential and entrapment efficiency were identified to critically effect the QTPPS (Figure 1). Prior knowledge gained through literature review and preliminary studies enabled to identify eight potential factors for evaluating experimental design as shown in Table 1.

\section{Screening by Plackett Burman design}

Plackett Burman design was applied for screening the most significant process and formulation factors to elucidate its effect on achieving the QTPP. The outline of the response on the dependent variable is shown in Table 4. The statistical evaluation with context to independent variable and its effect on the selected response is indicated in Table 5.

Particle size a very critical factor in design of inhalable nanoparticles, was seen to be affected by independent variables namely concentration of drug $\left(X_{1}\right)$, type of $\mathrm{GMO}\left(X_{2}\right)$, type of high HLB surfactant $\left(X_{4}\right)$, concentration of low HLB surfactant $\left(X_{5}\right)$,

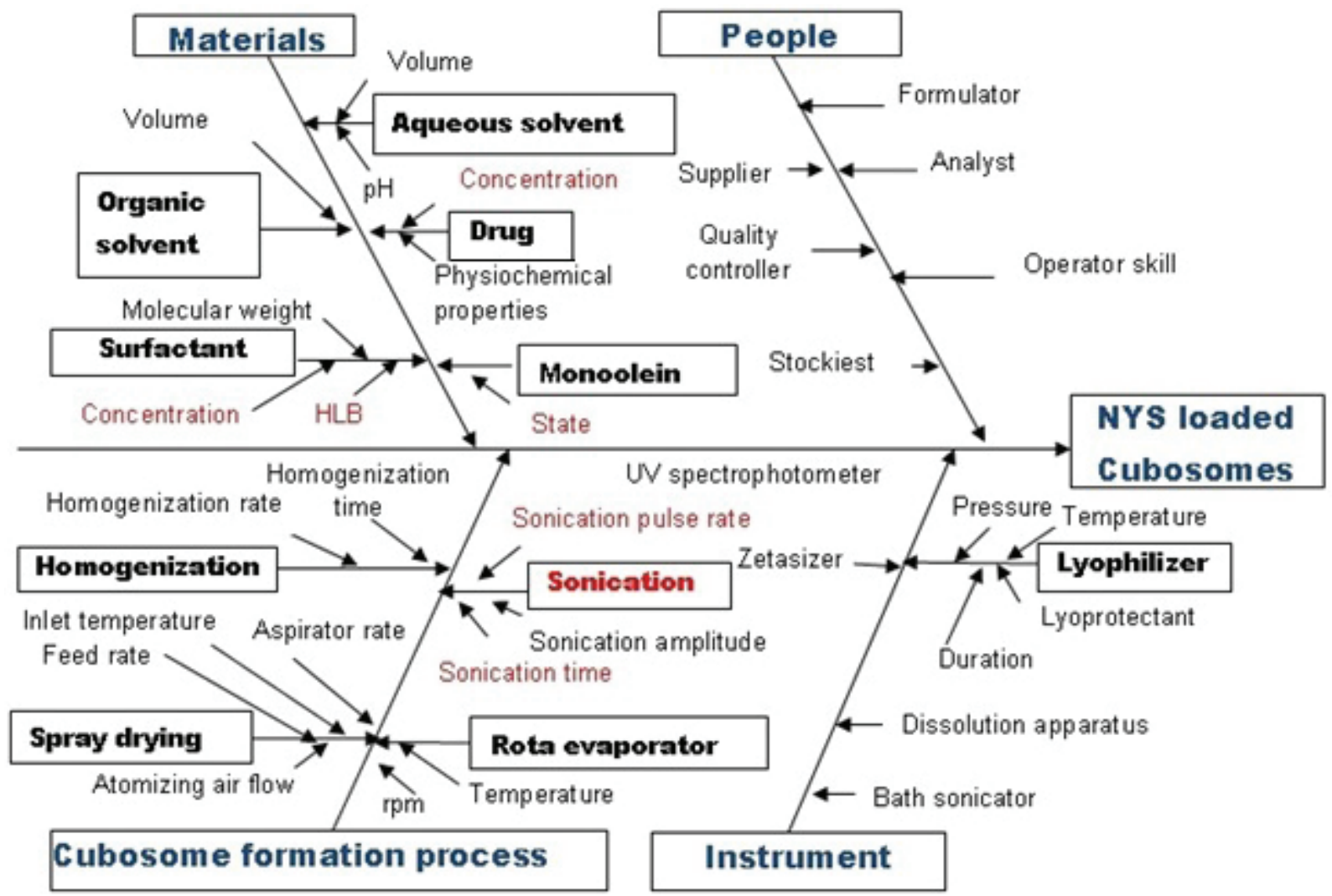

Figure 1. Ishikawa diagram representing process and formulation variables that may affect the CQAs of Cubosomes. The studied effect is "the fish head" and the potential causes and sub-causes define the "fish bone structure". 
Table 5. Outline of the response on the dependent variable obtained using Plackett Burman Design.

\begin{tabular}{|ccccc|}
\hline Formulation & $\begin{array}{c}\mathbf{Y}_{1} \text { : Average particle } \\
\text { size }(\mathrm{nm})\end{array}$ & $\begin{array}{c}\mathbf{Y}_{2} \text { : Polydispersity } \\
\text { index }(\mathrm{PDI})\end{array}$ & $\begin{array}{c}\mathbf{Y}_{3}: \text { Zeta potential } \\
(\mathrm{mV})\end{array}$ & $\begin{array}{c}\mathbf{Y}_{4} \text { : Drug Entrapment } \\
(\%)\end{array}$ \\
\hline D1 & 1638 & 0.376 & -13.4 & 82 \\
D2 & 505.5 & 0.568 & -25.4 & 84 \\
D3 & 2588 & 1 & -32 & 76 \\
D4 & 253 & 0.614 & -28.6 & 86 \\
D5 & 2687 & 1 & -18.6 & 79 \\
D6 & 3147 & 1 & -17.6 & 77 \\
D7 & 1328 & 0.376 & -27.9 & 76 \\
D8 & 347.7 & 0.446 & -27.3 & 83 \\
D9 & 878.6 & 0.833 & -30.9 & 78 \\
D10 & 1601 & 0.866 & -27.3 & 74 \\
D11 & 1069 & 0.697 & -14.1 & 76 \\
D12 & 817 & 0.62 & -33.5 & 82 \\
\hline
\end{tabular}

concentration of high HLB surfactant $\left(\mathrm{X}_{6}\right)$, sonication time $\left(\mathrm{X}_{7}\right)$ and pulse rate $\left(X_{8}\right)$. In a way $X_{2}, X_{4}, X_{6}$ and $X_{7}$ had a negative effect and decreased particle, while $X_{1}, X_{5}, X_{8}$ effected particle size positively by increasing particle size. Moreover literature reports have previously identified poloxamer to be 'gold standard surfactants as a stabilizer' for reducing particle size and for better stability profiling (Rizwan \& Boyd, 2015). We found that incorporation of higher grade of poloxamer (P-407) significantly reduced particle size. It was noted that, solid grade of GMO increased particle size in contrast to liquid grade of GMO. Although with increasing low HLB surfactant concentration particle size increased as it favored more structural binding with lipidic GMO (Dong, Chang, Qian, Tong, \& Zhou, 2016). Increase in sonication time lead to decrease in particle size and is credited to the high energy regions of cavitations and compression that grounds coarse dispersion into nanosize, the same has been reported elsewhere (Sugita, Ambarsari, \& Lidiniyah, 2015).

PDI of the colloidal dispersion was chosen to be CQA in order to obtain knowledge regarding the homogeneity of the particle size. The type of GMO $\left(X_{2}\right)$, type of high HLB surfactant $\left(X_{4}\right)$ and sonication time $\left(X_{7}\right)$ produced a decrease in $Y_{2}(P D I)$, whilst there is an increase in PDI observed with increase in concentration of drug $\left(\mathrm{X}_{1}\right)$, and concentration of low HLB surfactant $\left(\mathrm{X}_{5}\right)$.

Zeta potential $\left(\mathrm{Y}_{3}\right)$ of the system was also identified as CQA, to indicate physical stability of nanoparticle suspensions. $Y_{3,}$, notably depended on type of $G M O\left(X_{2}\right)$, type of high HLB surfactant $\left(X_{4}\right)$ and concentration of high HLB surfactant $\left(X_{6}\right)$ had a desirable effect whereas and sonication time $\left(X_{7}\right)$ had a negative effect.

Encapsulation efficiency was assigned to be CQA, since it is a paramount dependent variable that governs the delivery system, it manipulates the effectiveness of the nanoparticle to target site by increasing drug loading. Input factors such as concentration of drug $\left(X_{1}\right)$, concentration of high HLB surfac- tant $\left(X_{6}\right)$ and sonication time $\left(X_{7}\right)$ exhibited negative effects and increasing these input parameters lead to decline in drug entrapment efficiency, on the other hand factors such as type of GMO $\left(X_{2}\right)$, concentration of low HLB surfactant $\left(X_{5}\right)$, showcased a positive effect and improved encapsulation efficiency. Our findings are very well in concurrence with earlier reported outcomes, as the concentration of low HLB lipidic surfactant increases the payload also increases owing to addition of more solubilizing groups with expansion of the core radius (Hao et al., 2011). Increase in drug concentration lead to decrease in entrapment efficiency and could be as a result of insufficient quantity of lipidic polymers needed to effectively encapsulate the drug (Pawar, Gholap, Kuchekar, Bothiraja, \& Mali, 2015). Electrostatic repulsion in between surfactant and drug could be the possible reason for decline in EE\% with increase in concentration of high HLB surfactant. Plausible cause of decrease in $\mathrm{EE} \%$ with increase in sonication time is as a result of reduction in particle size during sonication cycle leading to loss of drug (Sugita et al., 2015). Thus, it becomes very essential to either fix certain independent variables or to optimize them to achieve the desired goal.

\section{Optimization using Box Beknkhn design}

Optimization of cubosomes was undertaken by fixing some of the independent variables. The concentration of drug was fixed to $1.23 \% \mathrm{w} / \mathrm{w}$ since our goal was to obtain particle size in the range $200-400 \mathrm{~nm}$ and maximize \% EE. Pulse rate was defined at 3/7 sec to achieve particle size in the desired range. Liquid grade of GMO, P-407 displayed better response for studied dependent variables, hence were fixed as type of GMO and type of high HLB surfactant respectively for BB design. Type of low HLB surfactant had insignificant effect on the selected responses, thus Span 83 was chosen. Input factors such as $X_{5}, X_{6}$ and $X_{7}$ were critically studied at three levels to optimize cubosomes, Table 6 depicts the observed responses. The coefficient of correlation and $p$ values is presented in Table 7. 
Table 6. Statistical Evaluation of the observed responses -Plackett Burman Design.

\begin{tabular}{|c|c|c|c|c|c|c|c|c|}
\hline \multirow{2}{*}{$\begin{array}{l}\text { Response } \\
\text { Source }\end{array}$} & \multicolumn{2}{|c|}{$\mathrm{Y}_{1}:$ Particle size } & \multicolumn{2}{|c|}{$Y_{2}:$ PDI } & \multicolumn{2}{|c|}{$Y_{3}$ : Zeta potential } & \multicolumn{2}{|c|}{$\mathrm{Y}_{4}$ : Entrapment efficiency } \\
\hline & Coefficient & $p$-value & Coefficient & $p$-value & Coefficient & $p$-value & Coefficient & $p$-value \\
\hline$a_{0}$ & 1404.98 & 0.0003 & 0.6997 & 0.0179 & -24.72 & 0.0181 & 79.42 & 0.0046 \\
\hline$x_{1}$ & 232.95 & 0.0003 & 0.0547 & 0.0330 & -1.28 & 0.0507 & -1.92 & 0.0019 \\
\hline $\mathrm{x}_{2}$ & -759.85 & $<0.0001$ & -0.0700 & 0.0205 & -1.92 & 0.0237 & 2.08 & 0.0016 \\
\hline$x_{3}$ & e & e & e & e & e & e & e & e \\
\hline$X_{4}$ & -249.20 & 0.0003 & -0.0460 & 0.0457 & -4.72 & 0.0040 & e & e \\
\hline$X_{5}$ & 277.05 & 0.0002 & 0.0727 & 0.0191 & -1.02 & 0.0773 & 0.7500 & 0.0121 \\
\hline$x_{6}$ & -102.13 & 0.0006 & e & e & -2.55 & 0.0136 & -0.5833 & 0.0198 \\
\hline$x_{7}$ & -71.95 & 0.0009 & -0.0408 & 0.0570 & 1.45 & 0.0404 & -1.08 & 0.0059 \\
\hline$x_{8}$ & 35.23 & 0.0018 & 0.0118 & 0.3653 & -0.4333 & 0.2860 & 0.3167 & 0.0507 \\
\hline
\end{tabular}

Table 7. Outline of the response on the dependent variable obtained using Box Behnken Design.

\begin{tabular}{|ccccc|}
\hline Formulation & $\mathbf{Y}_{1}$ : Particle size $(\mathrm{nm})$ & $\mathrm{Y}_{2}$ : Zeta potential $(\mathrm{mV})$ & $\mathbf{Y}_{3}$ : Polydispersity & $\mathbf{Y}_{\mathbf{4}}$ : Drug entrapment (\%) \\
\hline B1 & 197.7 & -10 & 0.459 & 76 \\
B2 & 209.5 & -18.5 & 0.360 & 78 \\
B3 & 263.5 & -14.4 & 0.283 & 82 \\
B4 & 383 & -26.02 & 0.149 & 86 \\
B5 & 191.7 & -9.98 & 0.387 & 78 \\
B6 & 338.7 & -27.5 & 0.222 & 88 \\
B7 & 188.6 & -8.81 & 0.525 & 76 \\
B8 & 175.7 & -6.33 & 0.410 & 81 \\
B9 & 233.5 & -18.1 & 0.154 & 87 \\
B10 & 171.8 & -4.77 & 0.217 & 86 \\
B11 & 229.8 & -14 & 0.245 & 88 \\
B12 & 310 & -31.3 & 0.320 & 90 \\
B13 & 188.6 & -8.34 & 0.568 & 77 \\
B14 & 185.7 & -8.43 & 0.462 & 78 \\
B15 & 320.4 & -29.2 & 0.317 & 90 \\
B16 & 420.8 & -27.3 & 0.362 & 92 \\
B17 & 169.3 & -4.11 & 0.333 & 70 \\
\hline
\end{tabular}

$R^{2}$ value for particle size $\left(Y_{1}\right)$, zeta potential $\left(Y_{2}\right)$, polydispersibility index $\left(Y_{3}\right)$, entrapment efficiency $\left(Y_{4}\right)$ is as $0.9457,0.8714$, 0.8775 and 0.9432 respectively. The $p$ values for all the model responses (Table 8 ) was less than 0.05 indicating that the concerning responses can be forecasted with exactness using the mathematical model.

The factors that impacted particle size significantly were concentration of low HLB surfactant $\left(X_{1}\right)$, concentration of high HLB surfactant $\left(X_{2}\right)$, sonication time $\left(X_{3}\right)$. Interaction of $X_{1} X_{2}$ and $X_{1} X_{3}$, quadratic effect of $X_{1}^{2}$ and $X_{3}^{2}$ significantly affected particle size. Increase in the concentration of low HLB surfactant and sonication time caused increase in particle size whereas increasing concentration of high HLB surfactant decreased particle size. Increase in the sonication time and concentration of low HLB surfactant exhibited synergistic action by promoting ionic interaction between drug and Span 83 leading to agglomerates of large particle size (Poddar \& Sawant, 2017). The polynomial equation of the second order to estimate mean particle size is as obtained:

$$
\begin{gathered}
Y=190.46+33.15 X_{1}-57.79 X_{2}+37.81 X_{3}-48.72 X_{1} X_{2}- \\
56.03 X_{1} X_{3}-12.80 X_{2} X_{3}+46.72 X_{1}^{2}+12.34 X_{2}^{2}+58.50 X_{3}^{2}
\end{gathered}
$$


Kazi and Dehghan. Development of inhalable cubosome nanoparticles of Nystatin for effective management of Invasive Pulmonary Aspergillosis

Table 8. Statistical Evaluation of the observed responses - Box Behnken Design.

\begin{tabular}{|c|c|c|c|c|c|c|c|c|}
\hline \multirow{2}{*}{$\begin{array}{c}\text { Response } \\
\text { Source }\end{array}$} & \multicolumn{2}{|c|}{$Y_{1}$ particle size $(\mathrm{nm})$} & \multicolumn{2}{|c|}{$\mathrm{Y}_{2}$ zeta potential (mV) } & \multicolumn{2}{|c|}{$\mathrm{Y}_{3}$ PDI } & \multicolumn{2}{|c|}{$Y_{4}$ Entrapment efficiency } \\
\hline & Coefficient & $p$-value & Coefficient & $p$-value & Coefficient & $p$-value & Coefficient & $p$-value \\
\hline$\beta_{0}$ & 190.46 & 0.0012 & -9.11 & 0.0091 & 0.48 & 0.0145 & 77.40 & 0.0015 \\
\hline$X_{1}$ & 33.15 & 0.0124 & 4.44 & 0.0125 & 0.0098 & 0.6716 & 4.25 & 0.0011 \\
\hline$x_{2}$ & -57.79 & 0.0006 & -5.26 & 0.0261 & 0.0011 & 0.9608 & -2.00 & 0.0407 \\
\hline$x_{3}$ & 37.81 & 0.0066 & 4.59 & 0.0228 & -0.058 & 0.0333 & 4.00 & 0.0015 \\
\hline$x_{1} x_{2}$ & -48.72 & 0.0104 & 3.71 & 0.1401 & -0.078 & 0.0422 & 0.0000 & 1 \\
\hline$x_{1} x_{3}$ & -56.03 & 0.0052 & 4.95 & 0.0620 & 0.047 & 0.1800 & -4.00 & 0.0094 \\
\hline$x_{2} x_{3}$ & -12.80 & 0.3919 & 3.37 & 0.1744 & -0.031 & 0.3507 & 1.0000 & 0.4052 \\
\hline$X_{1}^{2}$ & 46.72 & 0.0112 & 3.51 & 0.1508 & -0.076 & 0.0418 & 4.55 & 0.0044 \\
\hline$x_{2}^{2}$ & 12.34 & 0.3967 & -0.48 & 0.8325 & -0.096 & 0.0162 & 4.55 & 0.0044 \\
\hline$X_{3}{ }^{2}$ & 58.50 & 0.0037 & 10.04 & 0.0025 & -0.12 & 0.0046 & 2.05 & 0.1048 \\
\hline
\end{tabular}

\section{Table 9. The observed and predicted values of the optimized NYS cubosome formulation with respect to desirability}

\begin{tabular}{|ccc|}
\hline Response & Observed & Predicted \\
\hline Particle size $(\mathrm{nm})$ & 263.5 & 247.99 \\
Zeta potential (mV) & -14.4 & -14 \\
PDI & 0.283 & 0.278 \\
Entrapment efficiency (\%) & 82 & 81.81 \\
\hline
\end{tabular}

Zeta potential $\left(Y_{2}\right)$ of the colloidal dispersion dependent on individual factors $X_{1}, X_{2}, X_{3}$ in addition to quadratic effect of $X_{3}^{2}$ and had a significant on $Y_{2}$. The increase in concentration of P-407 and increase in sonication time affected zeta potential positively thus increased its magnitude while increase in concentration of Span 83 had negative impact on zeta potential by decreasing its magnitude. Similar outcomes have been portrayed previously (Sugita et al., 2015). The polynomial equation of the second order to estimate zeta potential is as obtained:

$$
\begin{gathered}
Y=-9.11+4.44 X_{1}-5.26 X_{2}-4.59 X_{3}+3.71 X_{1} X_{2}+4.95 X_{2} X_{3}+ \\
3.37 X_{2} X_{3}+3.51 X_{1}^{2}-0.48 X_{2}^{2}-10.04 \text { (Eq.4) }
\end{gathered}
$$

$\operatorname{PDI}\left(Y_{3}\right)$ was significantly affected by sonication time $\left(X_{3}\right)$, the interaction of $X_{1} X_{2}$ and the quadratic effect of individual inputs $\mathrm{X}_{1}{ }^{2}, \mathrm{X}_{2}{ }^{2}$ and $\mathrm{X}_{3}{ }^{2}$. Increasing the sonication time lead to decline in PDI values, similar findings of sonication time effecting PDI has been reported (Pawar et al., 2015). The polynomial equation of the second order to estimate PDI is as obtained:

$$
\begin{gathered}
Y=0.48+0.0098 X_{1}+0.0011 X_{2}-0.058 X_{3}-0.078 X_{1} X_{2}+0.047 X_{1} \\
X_{3}-0.031 X_{2} X_{3}-0.076 X_{1}^{2}-0.096 X_{2}^{2}-0.12 X_{3}^{2} \quad(\text { Eq.5) }
\end{gathered}
$$

Entrapment efficiency (\%EE) was influenced by $X_{1}, X_{2}, X_{3}, X_{1} X_{3}$ interaction, quadratic effect of $X_{1}{ }^{2}$ and $X_{2}{ }^{2}$, these terms showed significant effect. In case of $\%$ EE concentration of low HLB surfactant was critical variable and as the concentration increased entrapment also increased, similar findings have been reported (Gabr, Mortada, \& Sallam, 2017). However, concentration of Span 83 should be optimal so that the size of the nanoparticles is also beneath the defined dimensions. The polynomial equation of the second order to estimate \% EE is as obtained:

$$
\begin{gathered}
Y=77.40+ \\
4.25 X_{1}-2 X_{2}+4 X_{3}-4 X_{1} X_{2}+0 X X_{1} X_{2}+1 X_{2} X_{3}+ \\
4.55 X_{1}^{2}+4.55 X_{2}^{2}+2.05 X_{3}^{2} \quad \text { (Eq.6) }
\end{gathered}
$$

To carefully estimate the effect of various factors on selected responses, contour plots depicting the effect of two independent variables simultaneously (interactions) on the third variable (dependant variable) are presented in Figure 2.

The desirability function was obtained to acquire the optimized formulation. The optimum formulation was set on goal of achieving particle size less than $350 \mathrm{~nm}$, entrapment efficiency greater than 75\%, PDI less than 0.3 and zeta potential of upper limit -14 mV. Accordingly, cubosome nanoparticle with the projected levels of formulation factors was prepared to bear out the validity of the optimization method. Table 8 displays the optimum and predicted response of formulated optimized cubosome and Figure 3 is the representation of the overlaid contour plots.

\section{Polarised light microscopy (PLM)}

Crossed PLM image are presented in Figure 4. The PLM studies were undertaken for finding out whether the particles formed were cubosomes or hexosomes. The image appeared completely black under the cross polarizers indicating the presence of cubic phase, thus confirming formation of Cubosomes (Gabr et al., 2017).

\section{In-vitro antifungal activity}

In-vitro antifungal activity was estimated to confirm the efficacy of drug loaded cubosome against fungi of the genus Aspergillus fumigatus, the causative agent for IPA in immunocompromised patients. NYS solution and NYS suspension were considered as reference standards. The zone of inhibi- 


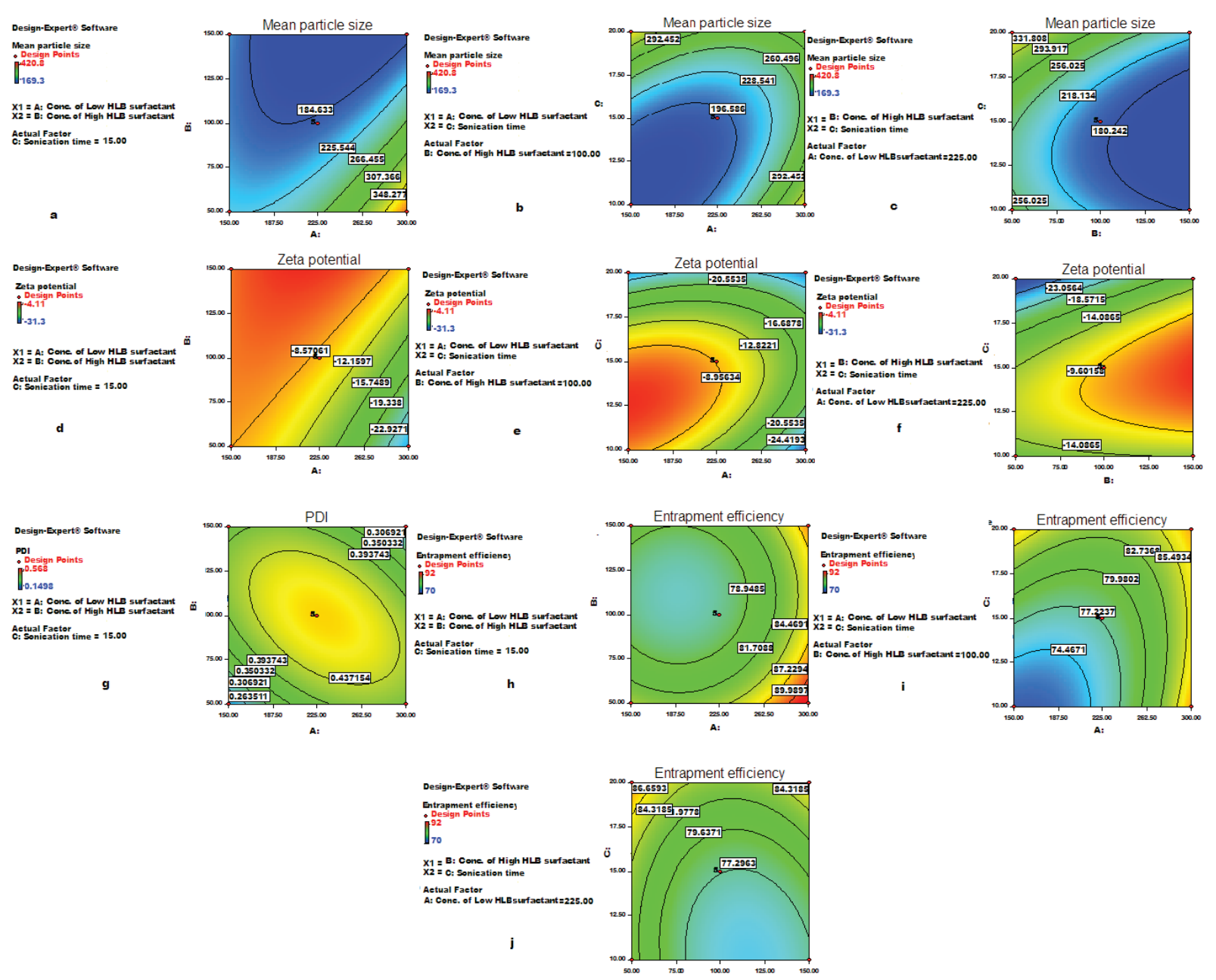

Figure 2. Contour plots presenting impact of (a) Concentration of low HLB surfactant and concentration of high HLB surfactant on mean particle size, (b) Concentration of low HLB surfactant and sonication time on mean particle size (c) Concentration of high HLB and sonication time on mean particle size (d) Concentration of low HLB surfactant and concentration of high HLB surfactant on zeta potential (e) Concentration of low HLB surfactant and sonication time on zeta potential ( $f$ ) Concentration of high HLB and sonication time on zeta potential ( $g$ ) Concentration of low HLB surfactant and concentration of high HLB surfactant on PDI(h) Concentration of low HLB surfactant and concentration of high HLB surfactant on entrapment efficiency (i) Concentration of low HLB surfactant and sonication time on entrapment efficiency (j) Concentration of high HLB and sonication time on entrapment efficiency.

tion of NYS from optimized cubosome (B3) was compared with NYS suspended in phosphate buffer and nystatin solution (Figure 5). It was notably seen that optimized NYS cubosomes displayed significantly higher zone of inhibition in comparison to NYS suspended in phosphate buffer $(p<0.05)$, whereas with pure drug solution no significant difference was found $(p>0.05)$.

\section{In-vitro drug release}

Release profile was investigated to assess the successful development and release of the encapsulated drug from cubosome nanoparticles. The study aimed to demonstrate the developed formulations superiority over drug suspension and drug solution. It was observed that complete drug release was achieved within 3 hours from NYS solution. The faster release of drug from pure solution is due to the fact that the drug particles are in molecular state. The release rate of NYS from optimized cubosome (B3) was further compared with NYS suspended in phosphate buffer (NYS suspension)
(Figure 6). Release profile points out to NYS release to be significantly higher from cubosome formulations compared to NYS suspension after $96 \mathrm{~h}$. Faster drug release from cubosome as compared to suspension can be attributed to the fact that the drug was in the solubilized state and moreover molecularly dispersed in amorphous form (Kassem, Mohsen, Samir Ahmed, \& Mohamed Essam, 2016; Prajapati, Jain, Jain, Sahu, \& Kohli, 2014). Although, in case of cubosome formulation a biphasic release pattern was seen, an initial burst release is due to adsorbed drug placed at or just below the surface of the nanoparticles followed by subsequent slower drug release rate up to $48 \mathrm{hrs}$. The results corroborate to earlier finding (Shi et al., 2017). The release kinetics from cubosome was studied using PCP DISSO V3 and was found to follow Higuchi release pattern $\left(R^{2}=0.941\right)$. Hence the release from these cubosome structures is controlled by the microstructure formed, number of water channels and the location of the drug moiety (Prajapati et al., 2014; Shi et al., 2017). 


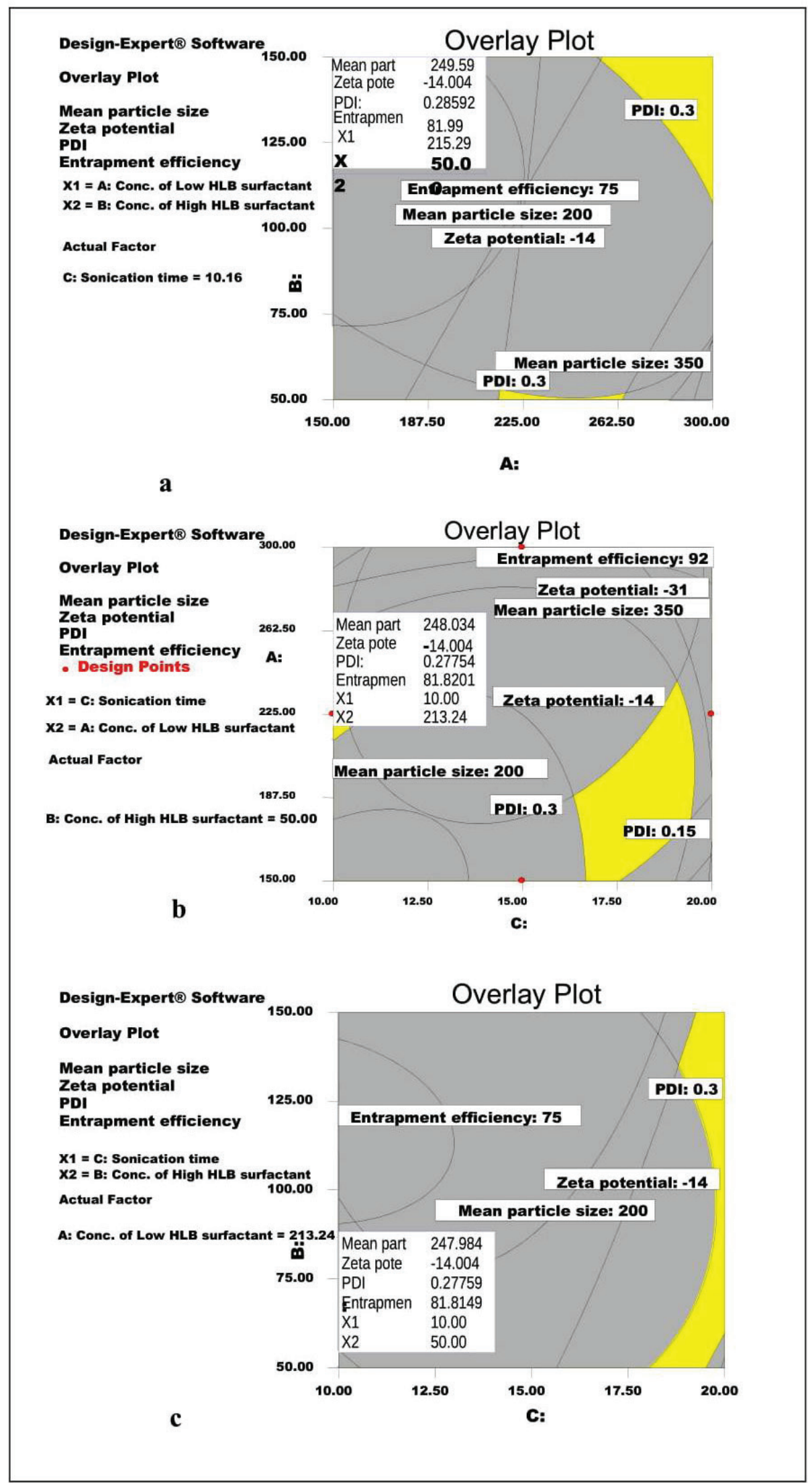

Figure 3. Design space for NYS cubosomes. Yellow regions depicts the likely permutations to achieve predictable results for average nanoparticle size (200-350 nm), zeta potential (<-14), encapsulation efficiency (>75\%) and polydispersity index (0.15-0.3). (a) Overlay plot of concentration of low HLB surfactant and concentration of high HLB surfactant, (b) overlay plot of concentration of low HLB surfactant and sonication time (c) overlay plot of concentration of high HLB surfactant and sonication time. 


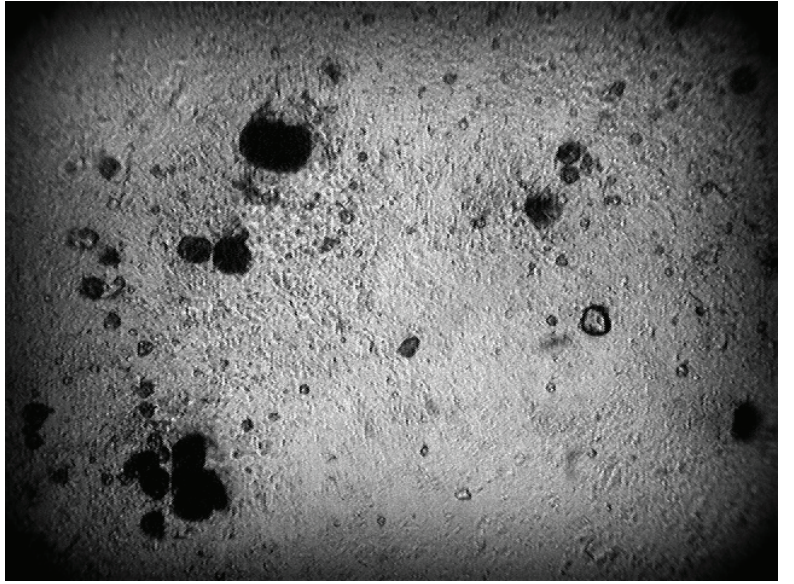

Figure 4. Polarised light microscopy image of optimized NYS cubosomes under cross polarizers.

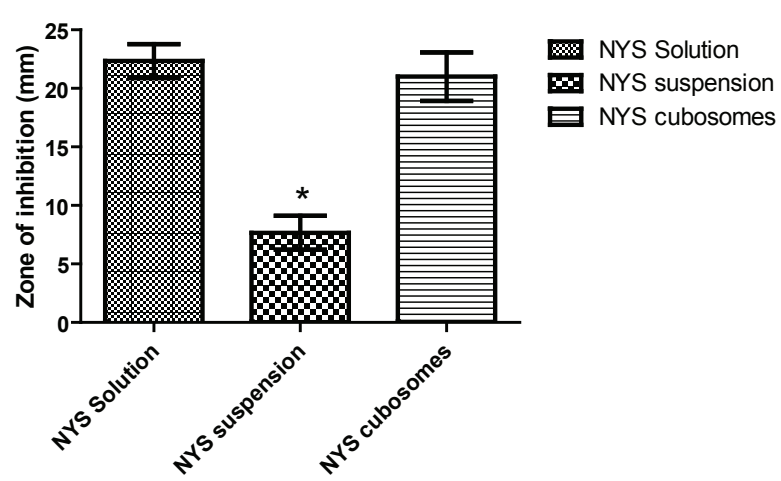

Figure 5. In-vitro antifungal activity of NYS solution, NYS Cubosomes and NYS suspension against Aspergillus fumigatus.

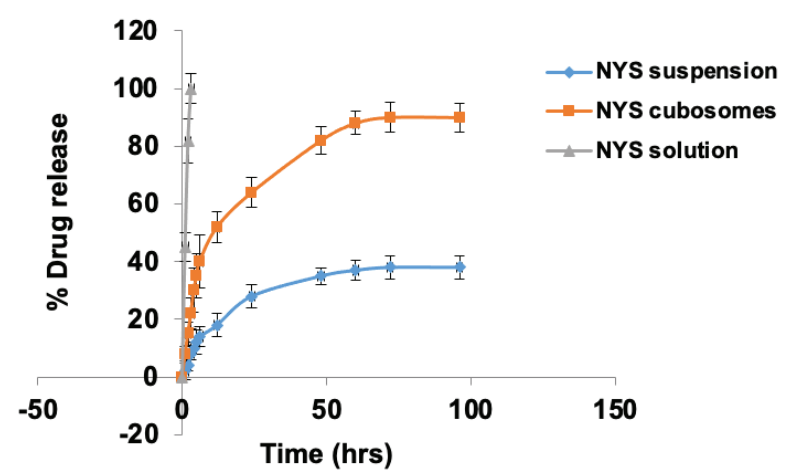

Figure 6. In-vitro drug release profile of NYS Cubosomes, NYS suspension and NYS solution.

\section{Cytotoxicity assays}

Cytotoxicity assay of NYS and optimized NYS cubosomes (B3) performed on A549 cell line after 72 hours of treatment is represented in Figure 7 . The cytotoxicity is expected to be maximum in solution since the drug is in its molecular state. The $\%$ cell viability on A549 cell line at concentration $100 \mu \mathrm{g} / \mathrm{ml}$ for these samples was found to be $22 \%$ and $70 \%$ respectively. $I C_{50}$ values were calculated to be $60.178 \mu \mathrm{g} / \mathrm{ml}$ and $151.35 \mu \mathrm{g} / \mathrm{ml}$ respectively. Thus the cubosome formulation exhibits reduced findings for nanocomposites have been reported elsewhere (Hussein-Al-Ali et al., 2014).

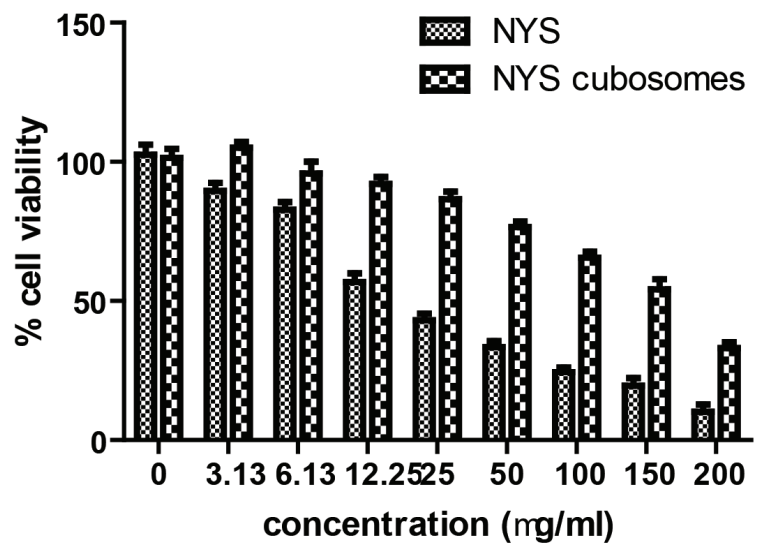

Figure 7. Cytotoxicity assay of NYS and NYS Cubosomes on A549 cell lines.

\section{In-vitro haemolytic assay}

To determine the toxic potential of the formulated cubosomes, in-vitro haemolytic assay was performed on various concentrations of both NYS and optimized formulation (B3). Figure 8 indicates that the $\mathrm{HC}_{50}$ for NYS cubosome was 400 $\mu \mathrm{g} / \mathrm{ml}$, while for NYS it was seen at $40 \mu \mathrm{g} / \mathrm{ml}$, similar findings have been reported (Kim et al., 2017). Thus, the results signify lower toxicity of the formulation as compared to NYS. This is observed as nystatin is well binded to the lipidic surfactant in the cubical structure, thus low release of free form is accessible to form dimer and induce haemolysis.

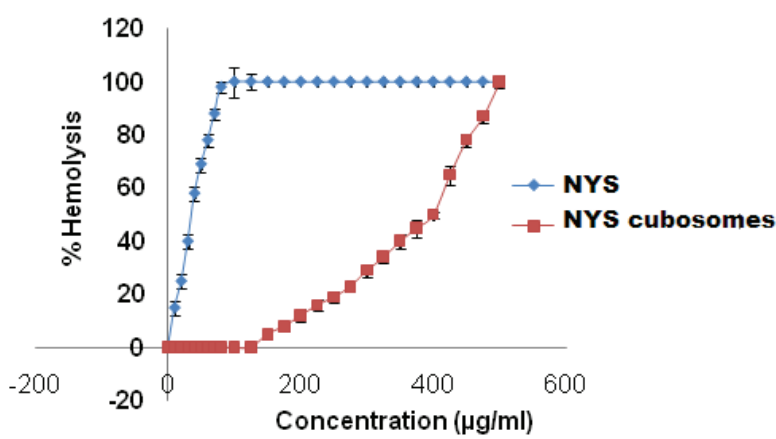

Figure 8. Hemolytic assay of NYS and NYS cubosomes.

\section{Small angle $\mathrm{X}$-ray scattering}

Scattering profile for the optimized formulation is presented in Figure 9. SAXS studies was undertaken to identify the cubic mesophasic structure, measure lattice parameters and also to confirm particle size of the formed nanoparticles. The scattering peaks ratios were indicative of $\mathrm{Pn} 3 \mathrm{~m}$ bicontinuous cubic phase $(\sqrt{ } 2: \sqrt{ } 3: \sqrt{ } 4)$ (Deshpande \& Singh, 2017). The lattice parameter was found to be $78.88 \mathrm{~nm}$ and the particle size was $245.77 \mathrm{~nm}$.

\section{Differential scanning calorimeter}

DSC thermograms of NYS, GMO, P-407 and NYS cubosomes are presented in Figure10. The thermogram of NYS exhibits 


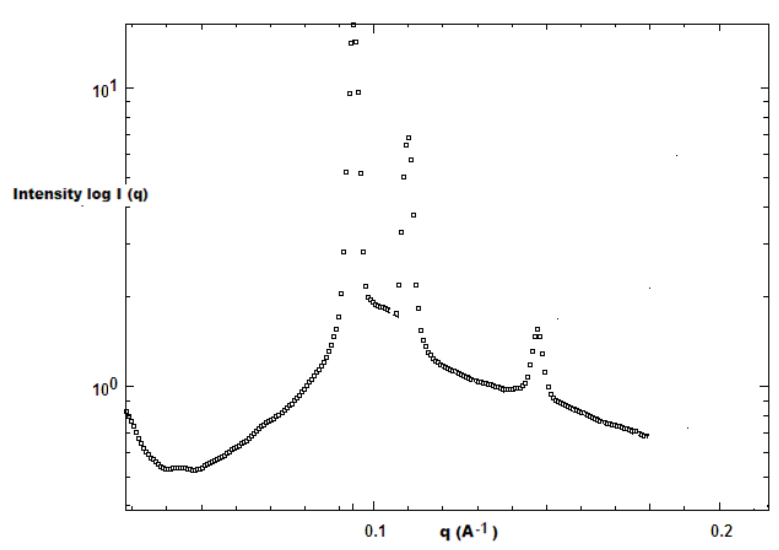

Figure 9. SAX scattering profile of optimized NYS Cubosomes.

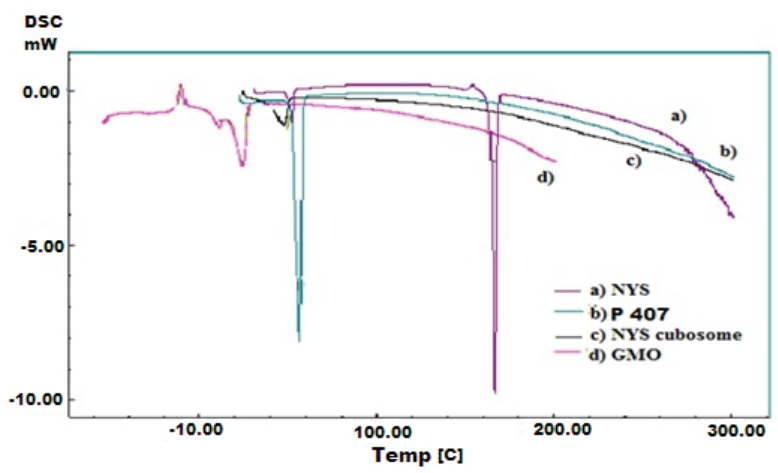

Figure 10. DSC thermogram of a) NYS b) P-407 c) NYS cubosome d) GMO.

a sharp endothermic melting peak at $166^{\circ} \mathrm{C}$ which is in conformity to literature reports (Michel, 1977). Nevertheless, NYS melting peak completely vanished in thermogram of NYS cubosomes. Thus, this justifies the incorporation of drug in the cubosomes to be a non-crystalline form.

\section{FTIR spectroscopy}

FTIR studies were undertaken to examine any interaction between NYS and excipients in the optimized cubosome formulation. Figure 11 illustrates the FTIR spectra of these samples. The spectra of NYS (Michel, 1977) distinctly shows a NH stretching peak at $3012 \mathrm{~cm}^{-1}, \mathrm{OH}$ stretching at $3351 \mathrm{~cm}^{-1}$, peak at 1701 $\mathrm{cm}^{-1}$ corresponds to unstrained lactone group, carboxylate ion

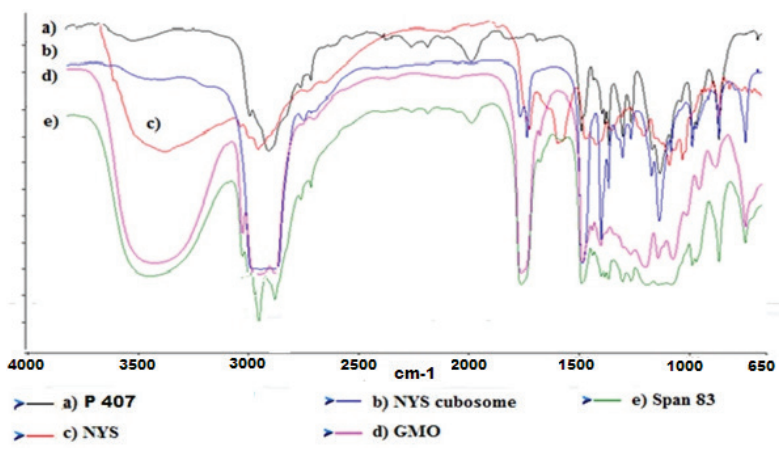

Figure 11. FTIR spectra of a) P-407 b) NYS cubosome c) NYS d) GMO e) Span 83. at $1574 \mathrm{~cm}^{-1}, \mathrm{CH}_{3}$ deformation (asym) and $\mathrm{CH}_{2}$ deformation at $1448 \mathrm{~cm}^{-1}, \mathrm{CH}_{3}$ deformation (sym) at $1399 \mathrm{~cm}^{-1}, \mathrm{C}-\mathrm{OH}$ stretching at $1069 \mathrm{~cm}^{-1}$ and $\mathrm{CH}$ deformation (out of plane) in $-\mathrm{CH}=\mathrm{CH}-$ (trans) at $946 \mathrm{~cm}^{-1}$. The IR spectra of $\mathrm{GMO}$ demonstrated $\mathrm{OH}$ stretching peak at $3400.80 \mathrm{~cm}^{-1}$. The $\mathrm{CH}_{2}$ stretching and ester bond was definite by peaks at $2960 \mathrm{~cm}^{-1}$ and $1740 \mathrm{~cm}^{-1}$ respectively. IR spectra of P-407 highlighted peaks at 2970.65 , 1343.17, and $1061.21 \mathrm{~cm}^{-1}$ related to $\mathrm{C}-\mathrm{H}$ aliphatic stretch, in plane O-H bond, and C-O stretch, respectively. Span 83 spectra showed characteristic peaks at $1630 \mathrm{~cm}^{-1}, 3430 \mathrm{~cm}^{-1}$, are assigned $\mathrm{OH}$ group. It is be noted, peaks at $1720 \mathrm{~cm}^{-1}, 1562 \mathrm{~cm}^{-1}$, $1357 \mathrm{~cm}^{-1}$ are attributed to the $\mathrm{C}=\mathrm{O}, \mathrm{C}-\mathrm{H}$ bending in $\mathrm{CH}_{3}$ and $\mathrm{CH}_{2}$. FTIR spectra for the drug loaded cubosome proves retention of characteristic functional groups, thus explicatory of no signs of interaction and assuring encapsulation of drug moiety in the nanostructure.

\section{X-ray diffraction}

XRD pattern of pure drug and optimized formulation is depicted in Figure 12. Typical sharp peaks for free NYS $2 \theta$ values 4.33, $13.95^{\circ}, 15.60^{\circ}, 16.72^{\circ}$ and $21.51^{\circ}$ (Michel, 1977) affirm to its crystalline nature. Though these peaks were not observed in the diffractogram of NYS cubosome formulation, thereby confirm to amorphous form of drug. DSC findings are in agreement to the above results and are suggestive of the drug encapsulated in the cubical nanostructure. However, the diffraction spectra of the optimized formulation did show some peaks at $2 \theta$ values $18.5^{\circ}$ and $22.37^{\circ}$, which may correspond to mannitol, the same has been reported (Sugita et al., 2015).

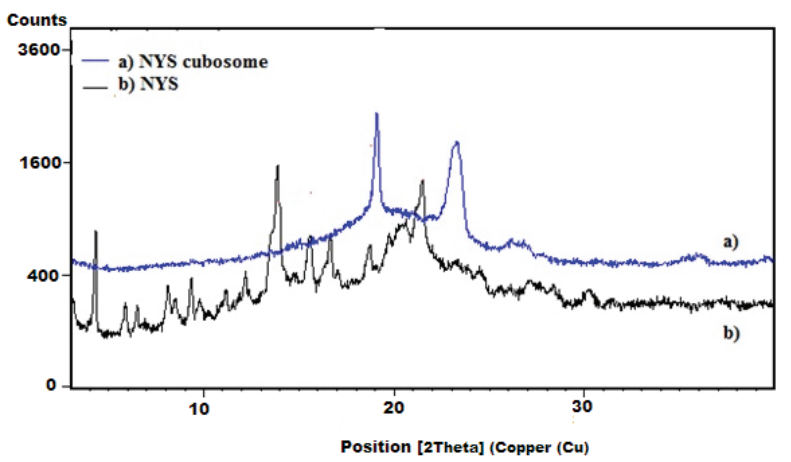

Figure 12. X-ray diffractogram of a) NYS cubosome b) NYS.

\section{FEG Transmission electron microscopy}

The structure of cubosomes is validated by both SAXS and TEM analysis. Figure 13 images denote outer shape and inner microstructure of the cubosomes. Outer virtually spherical structure with some irregular polyangular symmetry and extensive internal water channels clearly explains Pn3m mesophasic structure as interpreted through SAXS data and reported elsewhere (Shi et al., 2017). Particle size obtained by TEM (200 nm) is in good agreement to the zetasizer findings.

\section{CONCLUSIONS}

Nystatin loaded cubosome nanoparticles were formulated by dispersing the dry drug loaded lipidic film in phosphate buffer pH 7.4 by the aid of ultrasound probe sonicator. The effects of 


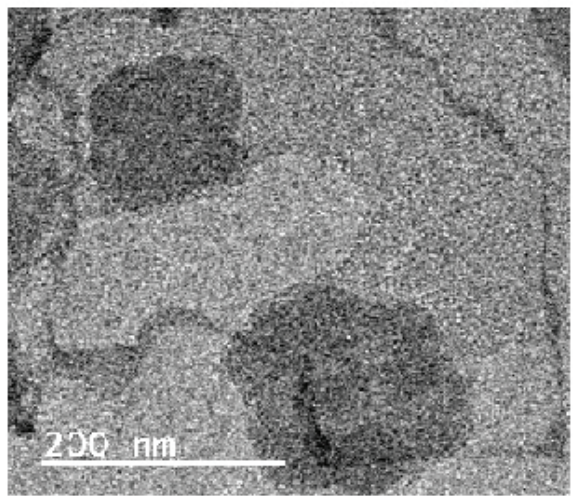

Figure 13. TEM images of optimized NYS cubosomes.

formulation and process parameters on CQAs of cubosome was screened and optimized with the help of experimental designs Placket-Burman followed by Box-Behnken. Design space was constructed following optimization of formulation. PLM, TEM images and SAXS profiles confirmed the formation of cubosome nanoparticles. XRD spectra, FTIR spectra and DSC thermogram indicated successful encapsulation of the drug. The in-vitro cell toxicity and in-vitro hemolytic assay revealed lower toxicity of NYS cubosomes as compared to free drug.

Although the in-vitro release profiles and in-vitro antifungal studies point out sustained effect, the conceptualization of target specificity can be assured only by evaluating the in-vivo deposition and fate of NYS-cubosomes in lungs which is essential for pulmonary administration of drugs. Thus, non-invasive feature and contemplated target specificity of nystatin loaded cubosome nanoparticles pave a way for its prospect as pulmonary delivery to combat IPA.

Peer-review: Externally peer-reviewed.

Author Contributions: Conception/Design of Study- M.K., M.H.D.; Data Acquisition- M.K.; Data Analysis/Interpretation- M.K., M.H.D.; Drafting Manuscript- M.K.; Critical Revision of Manuscript- M.K., M.H.D.; Final Approval and Accountability- M.K., M.H.D.; Technical or Material Support- M.K.; Supervision- M.H.D.

Conflict of Interest: The authors have no conflict of interest to declare.

Financial Disclosure: Authors declared no financial support.

Acknowledgments: The authors are thankful to Glenmark Pharmaceuticals (Mumbai, India) for providing Nystatin and Poloxamer P407, Dupont (Gurgaon, India) and Croda, (Mumbai, India) for generous supply of Dimodan HPM-90 and Cithrol GMO-HP-SO-LK respectively. We would also like to thank Cipla Limited (Mumbai, India) for providing Span 80 and Span 83.The authors thank SAIF, IIT-Bombay (Maharashtra, India) and Wockhardt Ltd. (Aurangabad, India) for rendering provisions to use SAXS facility and XRD, FTIR respectively.

\section{REFERENCES}

- $\quad$ Adhikari, K. (2017). In vitro Toxicity Study of Reconstituted Amphotericin B - Lipid Derivatives Dry Powder for Nebulization. Dhaka University Journal of Pharmaceutical Sciences, 15, 127.

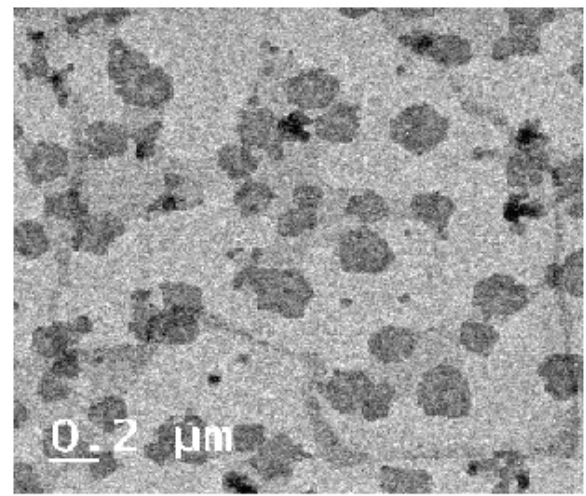

Ali, M. A., Noguchi, S., Iwao, Y., Oka, T., \& Itai, S. (2016). Preparation and Characterization of SN-38-Encapsulated Phytantriol Cubosomes Containing alpha-Monoglyceride Additives. Chemical \& Pharmaceutical Bulletin, 64(6), 577-584.

- $\quad$ Bei, D., Marszalek, J., \& Youan, B.-B. C. (2009). Formulation of dacarbazine-loaded cubosomes-part I: influence of formulation variables. AAPS PharmSciTech, 10(3), 1032-1039.

- $\quad$ Carneiro, S. P., Carvalho, K. V., de Oliveira Aguiar Soares, R. D., Carneiro, C. M., de Andrade, M. H. G., Duarte, R. S., \& Dos Santos, O. D. H. (2019). Functionalized rifampicin-loaded nanostructured lipid carriers enhance macrophages uptake and antimycobacterial activity. Colloids and Surfaces. B, Biointerfaces, 175, 306-313.

Chishti, N., Jagwani, S., Dhamecha, D., Jalalpure, S., \& Dehghan, M. H. (2019). Preparation, Optimization, and In Vivo Evaluation of Nanoparticle-Based Formulation for Pulmonary Delivery of Anticancer Drug. Medicina (Kaunas, Lithuania), 55(6).

- $\quad$ Chuealee, R., Aramwit, P., Noipha, K., \& Srichana, T. (2011). Bioactivity and toxicity studies of amphotericin B incorporated in liquid crystals. European Journal of Pharmaceutical Sciences: Official Journal of the European Federation for Pharmaceutical Sciences, 43(4), 308-317.

- Das, P. J., Paul, P., Mukherjee, B., Mazumder, B., Mondal, L., Baishya, R., Debnath, M. C., \& Dey, K. S. (2015). Pulmonary Delivery of Voriconazole Loaded Nanoparticles Providing a Prolonged Drug Level in Lungs: A Promise for Treating Fungal Infection. Molecular Pharmaceutics, 12(8), 2651-2664.

- Deshpande, S., \& Singh, N. (2017). Influence of Cubosome Surface Architecture on Its Cellular Uptake Mechanism. Langmuir, 33(14), 3509-3516.

- $\quad$ Dong, Y., Chang, Y., Qian, W., Tong, J., \& Zhou, J. (2016). Effects of surfactants on size and structure of amylose nanoparticles prepared by precipitation. Bulletin of Materials Science, 39.

- El-Ridy, M. S., Abdelbary, A., Essam, T., El-Salam, R. M. A., \& Kassem, A. A. A. (2011). Niosomes as a potential drug delivery system for increasing the efficacy and safety of nystatin. Drug Development and Industrial Pharmacy, 37(12), 1491-1508.

- $\quad$ Esposito, E., Cortesi, R., Drechsler, M., Paccamiccio, L., Mariani, P., Contado, C., Stellin, E., Menegatti, E., Bonina, F., \& Puglia, C. (2005). Cubosome dispersions as delivery systems for percutaneous administration of indomethacin. Pharmaceutical Research, 22(12), 2163-2173. Kim, E. S., Han, K. B., Lin, S., Kong, D., Bai, L., Deng, Z., ... \& Lee, M. J. (2017). Polyene compound, method for preparing the same, and antifungal drug comprising novel polyene compound as active ingredient U.S. Patent No. 9,605,015. Washington, DC: U.S. Patent and Trademark Office.

- Fernandez Campos, F., Calpena Campmany, A. C., Rodriguez Delgado, G., Lopez Serrano, O., \& Clares Naveros, B. (2012). Development and characterization of a novel nystatin-loaded nanoemulsion for the buccal treatment of candidosis: ultrastructural effects and release studies. Journal of Pharmaceutical Sciences, 101(10), 3739-3752. 
- $\quad$ Furedi, P., Papay, Z. E., Kovacs, K., Kiss, B. D., Ludanyi, K., Antal, I., \& Klebovich, I. (2017). Development and characterization of the voriconazole loaded lipid-based nanoparticles. Journal of Pharmaceutical and Biomedical Analysis, 132, 184-189.

- Gabr, M. M., Mortada, S. M., \& Sallam, M. A. (2017). Hexagonal Liquid Crystalline Nanodispersions Proven Superiority for Enhanced Oral Delivery of Rosuvastatin: In Vitro Characterization and In Vivo Pharmacokinetic Study. Journal of Pharmaceutical Sciences, 106(10), 3103-3112.

- Hao, J., Fang, X., Zhou, Y., Wang, J., Guo, F., Li, F., \& Peng, X. (2011). Development and optimization of solid lipid nanoparticle formulation for ophthalmic delivery of chloramphenicol using a Box-Behnken design. International Journal of Nanomedicine, 6, 683-692.

- Hussein-Al-Ali, S. H., El Zowalaty, M. E., Kura, A. U., Geilich, B. Fakurazi, S., Webster, T. J., \& Hussein, M. Z. (2014). Antimicrobial and controlled release studies of a novel nystatin conjugated iron oxide nanocomposite. BioMed Research International, 2014, 651831.

- Islam, N., \& Ferro, V. (2016). Recent advances in chitosan-based nanoparticulate pulmonary drug delivery. Nanoscale, 8(30), 14341-14358.

- Kassem, A., Mohsen, A., Samir Ahmed, R., \& Mohamed Essam, T. (2016). Self-nanoemulsifying drug delivery system (SNEDDS) with enhanced solubilization of nystatin for treatment of oral candidiasis: Design, optimization, in vitro and in vivo evaluation. Journal of Molecular Liquids, 218, 219-232.

- Khan, M. A., Faisal, S. M., \& Mohammad, O. (2006). Safety, efficacy and pharmacokinetics of tuftsin-loaded nystatin liposomes in murine model. Journal of Drug Targeting, 14(4), 233-241.

- $\quad$ Kim, H.-J., Son, J. S., \& Kwon, T.-Y. (2018). Antifungal Effect of a Dental Tissue Conditioner Containing Nystatin-Loaded Alginate Microparticles. Journal of Nanoscience and Nanotechnology, 18(2), 848-852.

- Kosmidis, C., \& Muldoon, E. G. (2017). Challenges in the management of chronic pulmonary aspergillosis. Medical Mycology, 55(1), 63-68.

- $\quad$ Lestner, J. M., Howard, S. J., Goodwin, J., Gregson, L., Majithiya, J., Walsh, T. J., Jensen, G. M., \& Hope, W. W. (2010). Pharmacokinetics and pharmacodynamics of amphotericin B deoxycholate, liposomal amphotericin B, and amphotericin B lipid complex in an in vitro model of invasive pulmonary aspergillosis. Antimicrobial Agents and Chemotherapy, 54(8), 3432-3441.

- Madheswaran, T., Kandasamy, M., Bose, R. J., \& Karuppagounder, V. (2019). Current potential and challenges in the advances of liquid crystalline nanoparticles as drug delivery systems. Drug Discovery Today, 24(7), 1405-1412.

- Mandal, S., Prathipati, P. K., Belshan, M., \& Destache, C. J. (2019). A potential long-acting bictegravir loaded nano-drug delivery system for HIV-1 infection: A proof-of-concept study. Antiviral Research, 167, 83-88.

- Maqsood, I., Masood, M. I., Bashir, S., Nawaz, H. M. A., Anjum, A. A., Shahzadi, I., Ahmad, M., \& Imran Masood, I. M. (2015). Preparation and in vitro evaluation of Nystatin micro emulsion based gel. Pakistan Journal of Pharmaceutical Sciences, 28(5), 1587-1593.

- Marin-Quintero, D., Fernandez-Campos, F., Calpena-Campmany, A. C., Montes-Lopez, M. J., Clares-Naveros, B., \& Del Pozo-Carrascosa, A. (2013). Formulation design and optimization for the improvement of nystatin-loaded lipid intravenous emulsion. Journal of Pharmaceutical Sciences, 102(11), 4015-4023.

- Michel, G. W. (1977). Nystatin (K. Florey (ed.); Vol. 6, pp. 341-421). Academic Press.

- Newman, S. P. (2017). Drug delivery to the lungs: challenges and opportunities. Therapeutic Delivery, 8(8), 647-661.
Offner, F., Krcmery, V., Boogaerts, M., Doyen, C., Engelhard, D., Ribaud, P., Cordonnier, C., de Pauw, B., Durrant, S., Marie, J.-P., Moreau, P., Guiot, H., Samonis, G., Sylvester, R., \& Herbrecht, R. (2004). Liposomal nystatin in patients with invasive aspergillosis refractory to or intolerant of amphotericin B. Antimicrobial Agents and Chemotherapy, 48(12), 4808-4812.

- Pawar, A. P., Gholap, A. P., Kuchekar, A. B., Bothiraja, C., \& Mali, A. J. (2015). Formulation and evaluation of optimized oxybenzone microsponge gel for topical delivery. Journal of Drug Delivery, 2015, 261068. Petrikkou, E., Rodriguez-Tudela, J. L., Cuenca-Estrella, M., Gomez, A., Molleja, A., \& Mellado, E. (2001). Inoculum standardization for antifungal susceptibility testing of filamentous fungi pathogenic for humans. Journal of Clinical Microbiology, 39(4), 1345-1347.

- $\quad$ Pinto Reis, C., Vasques Roque, L., Baptista, M., \& Rijo, P. (2016). Innovative formulation of nystatin particulate systems in toothpaste for candidiasis treatment. Pharmaceutical Development and Technology, 21(3), 282-287.

- Poddar, A., \& Sawant, K. (2017). Optimization of Galantamine Loaded Bovine Serum Albumin Nanoparticles by Quality by Design and Its Preliminary Characterizations. J Nanomed Nanotechnol, 8(5), 1-10.

- $\quad$ Prajapati, V., Jain, A., Jain, R., Sahu, S., \& Kohli, D. V. (2014). Treatment of cutaneous candidiasis through fluconazole encapsulated cubosomes. Drug Delivery and Translational Research, 4(5-6), 400-408.

Rani, S., Gothwal, A., Pandey, P. K., Chauhan, D. S., Pachouri, P. K., Gupta, U. D., \& Gupta, U. (2018). HPMA-PLGA Based Nanoparticles for Effective In Vitro Delivery of Rifampicin. Pharmaceutical Research, 36(1), 19.

Rizwan, S. B., Assmus, D., Boehnke, A., Hanley, T., Boyd, B. J., Rades, T., \& Hook, S. (2011). Preparation of phytantriol cubosomes by solvent precursor dilution for the delivery of protein vaccines. European Journal of Pharmaceutics and Biopharmaceutics: Official Journal of Arbeitsgemeinschaft Fur Pharmazeutische Verfahrenstechnik e.V, 79(1), 15-22.

Rizwan, S.??, \& Boyd, B. (2015). Cubosomes: Structure, Preparation and Use as an Antigen Delivery System (pp. 125-140).

Ryder, N. S. (1999). Antifungal agents. IDrugs: The Investigational Drugs Journal, 2(12), 1253-1255.

- $\quad$ Sakeer, K., Al-Zein, H., Hassan, I., Desai, S., \& Nokhodchi, A. (2010). Enhancement of dissolution of nystatin from buccoadhesive tablets containing various surfactants and a solid dispersion formulation. Archives of Pharmacal Research, 33(11), 1771-1779.

Shi, X., Peng, T., Huang, Y., Mei, L., Gu, Y., Huang, J., Han, K., Li, G., Hu, C., Pan, X., \& Wu, C. (2017). Comparative studies on glycerol monooleate- and phytantriol-based cubosomes containing oridonin in vitro and in vivo. Pharmaceutical Development and Technology, 22(3), 322-329.

Silva, F. C., Marto, J. M., Salgado, A., Machado, P., Silva, A. N., \& Almeida, A. J. (2017). Nystatin and lidocaine pastilles for the local treatment of oral mucositis. Pharmaceutical Development and Technology, 22(2), 266-274.

Sugita, P., Ambarsari, L., \& Lidiniyah. (2015). Optimization of Ketoprofen-loaded Chitosan Nanoparticle Ultrasonication Process. Procedia Chemistry, 16, 673-680.

Szalewski, D. A., Hinrichs, V. S., Zinniel, D. K., \& Barletta, R. G. (2018). The pathogenicity of Aspergillus fumigatus, drug resistance, and nanoparticle delivery. Canadian Journal of Microbiology, 64(7), 439-453.

- von Halling Laier, C., Gibson, B., van de Weert, M., Boyd, B. J., Rades, T., Boisen, A., Hook, S., \& Nielsen, L. H. (2018). Spray dried cubosomes with ovalbumin and Quil-A as a nanoparticulate dry powder vaccine formulation. International Journal of Pharmaceutics, 550(1-2), 35-44. Yang, Z., Tan, Y., Chen, M., Dian, L., Shan, Z., Peng, X., \&Wu, C. (2012). Development of amphotericin B-loaded cubosomes through the SolEmuls technology for enhancing the oral bioavailability. AAPS PharmSciTech, 13(4), 1483-1491. 